\title{
THE IMPORTANCE OF INTEGRATED AND
}

RESOLUTIVE CARE TO REDUCE THE TIME TO

START TREATMENT IN 8,357 BREAST CANCER

PATIENTS TREATED BY THE BRAZILIAN UNIFIED

HEALTH SYSTEM (SUS) AT A WOMEN'S HEALTH

REFERENCE CENTER (CRSM).

Igor Duarte de Oliveira', Jorge Yoshinori Shida¹, André Mattar¹, Silvana Maria Graziani', Luiz Henrique Gebrim¹

${ }^{1}$ Hospital Pérola Byington, Centro de Referência da Saúde da Mulher-São Paulo (SP), Brazil.

Introduction: In 2021, it is estimated that Brazil will have 66,280 new cases of breast cancer. Unfortunately, around 55\% of the cases treated by the Brazilian Unified Health System (SUS) are symptomatic and are in advanced stages. The long time before biopsy and the late start of treatment contribute to the progression of these tumors, with worsening prognosis. In order to reduce the time to start therapy, we implemented a model of integrated multidisciplinar care with high resolution (CARE). Objectives: The purpose of this work is to demonstrate the main barriers and the solutions found to reduce the time of treatment start at the institution, according to Law no. 12,732, which recommends the start of therapy within 60 days of diagnosis, preventing the progression of the disease and reducing mortality. Methods: The information was registered at the Women's Health Reference Center (CRSM) data colletion system from the first day of care until the start of therapy (surgery or chemotherapy), in a cross-sectional cohort of 8,357 patients from January 2011 to December 2018. Patients referred were seen at CARE and underwent consultation with an anesthesiologist or oncologist in the service having a previous appointment to start treatment. Results: After the integration of anestheologists and clinical oncologists, in 2014, there was a significant reduction (from 55 to 38 days) in the time of treatment start. Discussion: CARE started integrated care with biopsy on the same day as the initial consulation in 2006, reducing returns and time to diagnosis from 90 to 15 days. CRSM serves more than 1,200 new cases/year, about 30\% of SUS cases in the city of São Paulo (SP). A 12-week surgical delay for all breast cancer patients from one year (for example in the COVID 19 pandemic) would lead to 1,400 excess deaths in the United Kingdon and 6,100 in the United States, assuming surgery was the first choice of treatment for $83 \%$ of the patients. Conclusion: Our experience shows that the training of mastologists to perform a biopsy in the first consultation, that the public/private partnership to obtain the anatomopathological and immunohistochemical results in 15 days and that a multidisciplinary integrated care is possible, allowing the average onset of treatment to be close to 30 days, which may reduce the proportion of advanced cases in SUS. 